\title{
XXXIII. Simple method for calibrating thermometers
}

\section{Silas W. Holman}

To cite this article: Silas W. Holman (1882) XXXIII. Simple method for calibrating thermometers, Philosophical Magazine Series 5, 14:88, 294-299, DOI:

10.1080/14786448208627267

To link to this article: http://dx.doi.org/10.1080/14786448208627267

曲 Published online: 08 Jun 2010.

Submit your article to this journal ¿

Џ Article views: 2

Q View related articles $₫$ 
least resistance-will combine to carry the luminous particles emitted from the comet in advance of the mass.

That the change in the direction of a comet's tail should take place with the rapidity and to the extent observed in the case of the enormous appendages of some of these bodies may still appear surprising. But it must be remembered that, in these cases, we can speak only of what we see. The conical mass of gases or vapours extending behind the nucleus of a comet may attain, in the case of the largest of these bodies, to an expansion much greater than the part visible, which may consist only of the parts that receive the strongest impulses from the centre of force; so that when the tail seems to have swung round through an enormous are in the sky, what has really happened may be only that the line along which the substances forming it become visible may have shifted, in consequence of the direction of the impulses proceeding from the head having altered.

As the time when the most rapid alteration in the direction of the tail of a comet takes place necessarily coincides with that when the expansive action of the sun on the substances emitted from the comet is at its maximum, there must be the less difficulty in admitting the last hypothesis as an explanation of this phenomenon. It is the only one, so far as I see, that offers any difficulty in the way of the theory now proposed respecting the tails of comets, which may be summed up in the proposition that, as the incandescence of meteoric bodies proves to us the existence of a widely diffused atmosphere surrounding the earth, so the development of the tails of Comets proves to us the existence of a much more widely diffused atmosphere surrounding the sun-both sets of phenomena being due to the same cause, namely the resistance of these atmospheres to bodies rapidly passing through them.

I am yours \&c.,

E. Vanstttart Neale.

15 Portsmouth Street, Manchester, August 21, 1882.

XXXIII. Simple Method for Calibrating Thermometers. By Silas W. Holman*.

THE calibration of a thermometer by most of the methods 1 in ordinary use is a tedious and somewhat difficult operation, and hence often neglected even in important work. For the purpose of supplying a method simple both in observation and computation, and at the same time accurate, the following

* From Silliman's American Journal, No, 136, p. 278. 
process is described, which, although involving little that is novel, has not to my knowledge been used before.

First, however, it is necessary to recall to the attention of observers the fact that, without calibration-correction, the readings of a thermometer having a scale of equal linear parts cannot be relied upon within one or more divisions of this scale, and that thermometer-makers, knowing this, almost miversally space the graduation upon the tube to correspond more or less closely with the shape of the bore, as determined by previous calibration, or by comparison with a standard (!) instrument. This practice is much more general than is ordinarily supposed, and has an important bearing upon the accuracy of the work done with such instruments. For the scale thus made is merely approximate, the dividing-engine or other tool being usually changed only at such intervals as to make the average error less than some specified amount. An inspection of these conditions will show that the calibration of such a tube and scale can be only approximate, except with corrections for the inequalities of the spacing, involving an amount of labour disproportionate to the result obtained. The best makers, such as Fastré, Baudin, and others, have produced satisfactory thermometers graduated to equal volumes; but even these are not as reliable as instruments of less cost with a scale of equal linear parts, say of millimetres, supplemented by a calibration by the observer. The best form of tube for almost all work is one backed with white enamel, with an inverted pear-shaped bulb at the upper end of the capillary (a very important feature), and with a scale of equal arbitrary linear parts $(0.7$ to 1 millim. is a suitable length for estimation of tenths) or of approximate degrees, for convenience, etched or engraved upon it.

Without reviewing here the methods proposed by various writers, it may be said that it has been the general plan to select beforehand upon the scale two points between which to make the calibration, this space being the "calibration unit," the errors of these points being, of course, zero. This plan bas led to unnecessary complexity. Such an assumption is no more requisite in calibration after a scale has been put upon the tube, than in calibrating by the dividing-engine or micrometer before making the scale. It is obvious that the selec-. tion of these points is wholly arbitrary, and, if used at all, one or both of them may, if desirable, be chosen after the observations with the calibrating-thread have been made. The choice should be made with a riew to facilitating the work. Hence the use of the observed freezing- and boiling-points, upon which some methods are based, is most undesirable. 
In the method which will now be given, either one or both of these points may be left to be selected, according to the combined conditions of length of thread employed, shape of the tube, and numerical convenience, after the observations with the thread have been made.

Let it be desired to find the calibration-corrections for a given tube. Determinations which will give the errors of every 3 centim. of length will ordinarily be sufficient; but this must depend on the result sought. Separate a thread of mercury of about that length. The actual length of the thread within two or three millimetres is of no consequence whatever; and hence a suitable thread can be obtained in a very short time.

Set the thread with its lower end at or near the beginning of the graduation: call the reading* of the lower end of the thread $l_{1}$, and that of the upper end $u_{1}$. Move the thread less than 1 millim. and read again, finding thus $l_{2}$ and $u_{2}$. Move the thread about 1 centim., and read $l_{3}$ and $u_{3}$. Move the thread less than 1 millim., and read $l_{4}$ and $u_{4}$. So continue throughout the whole length of graduation, increasing the number of settings or repeating the whole series in reverse order and several times, if the highest attainable precision is desired. This alternation between 1 millim. and 1 centim. in setting tends towards the better elimination of errors in estimation. It is not, however, essential, nor even always as well as an equal number of distributed readings. This must depend upon the skill of the observer. Avoid, as far as convenient, taking readings with an end of the thread apparently just at the line of the scale, as the width of the line, even in the best scales, is a source of considerable error $\uparrow$. If any point $(e . g$. the zero-point of the graduation) has for any reason been selected as the first of which the error should be assumed zero, the settings may to advantage, though not necessarily, be made to extend each way from this.

Then $u_{1}-l_{1}, u_{2}-l_{2}, \& c$. will give a series of lengths of the calibrating-thread in all parts of the tube. Before reuniting this thread to the rest of the mercury, plot points with abscissas $l_{1}, l_{2}, \& c$. , and ordinates $u_{1}-l_{1}, u_{2}-l_{2}$, \&c., the corresponding lengths of thread, and draw a smooth curve through the points thus obtained. This line will give a general idea of the form of the capillary bore; and should any parts of it show considerable irregularities, the corresponding portions of the tube should at once be reexplored with the thread.

If not already done, the point $A$ upon the scale, to be used

* Tenths of a division are supposed to be read by estimation.

$\uparrow$ Some of the advantages of Neumann's method are offset by this error. 
as the starting- or reference-point of the computation, should now be selected. In general the extreme ends of the tube are to be avoided, as more likely to have been rendered irregular or rapidly tapering in the process of making or joining on the bulbs. If the zero of the numbering is placed one or two centimetres from the bottom of the tube, it forms a desirable starting-point.

Find upon the curre the ordinate $u^{\prime}$ corresponding to the abscissa $\mathrm{A}$; then with abscissa $\mathrm{A}+u^{\prime}$ find the corresponding ordinate $u^{\prime \prime}$, with abscissa $\mathrm{A}+u^{\prime}+u^{\prime \prime}$ find the corresponding ordinate $u^{\prime \prime \prime}$, continuing to the upper limit of the graduation. If $A$ is at a sufficient distance from the lower end of the graduation, find a similar series below the point $A$. These points, $\mathrm{A}, \mathrm{A}+u^{\prime}, \mathrm{A}+u^{\prime}+u^{\prime \prime}, \& c$. , upon the graduation are separated by equal volumes of the capillary. Select any one of these as the second point of which the error is to be arbitrarily assumed as zero, and call this $B$. Then

$$
\mathrm{A}+u^{\prime}+u^{\prime \prime}+\ldots+u \mathrm{nth}=\mathrm{B} \text {. }
$$

There are thus $n$ spaces of equal volumie between $A$ and $B$; and these correspond each to $\frac{1}{n}$ th of the interval $\mathrm{B}-\mathrm{A}$. Hence the true reading (which, however, it is not necessary to compute numerically) at the point

$$
\begin{aligned}
& \mathrm{A} \text { is } \mathrm{A} \text {, } \\
& \mathrm{A}+u^{\prime} \quad \text { is } \mathrm{A}+\frac{1}{n}(\mathrm{~B}-\mathrm{A}) \text {, } \\
& \mathrm{A}+u^{\prime}+u^{\prime \prime} \text { is } \mathrm{A}+\frac{2}{n}(\mathrm{~B}-\mathrm{A}) \text {, } \\
& B \text { is } B \text {. }
\end{aligned}
$$

And the error obtained by subtracting the true readings, as given in the right-hand column, from the corresponding actual readings, given in the left-hand column, at

$$
\begin{aligned}
& \text { A is } 0 \text {, } \\
& \mathrm{A}+u^{\prime} \quad \text { is } \mathrm{A}+u^{\prime}\left\{\mathrm{A}+\frac{1}{n}(\mathrm{~B}-\mathrm{A})\right\}=u^{\prime}-\frac{1}{n}(\mathrm{~B}-\mathrm{A}) \text {, } \\
& \mathrm{A}+u^{\prime}+u^{\prime \prime} \text { is } u^{\prime} u^{\prime \prime}-+\frac{2}{n}(\mathrm{~B}-\mathrm{A}) \text {, } \\
& B \text { is } 0 \text {. }
\end{aligned}
$$

In selecting $B$ it might have been assumed equal to $A+u^{\prime}$, thus making $n=1$. This would somewhat simplify the calcu- 
lation, and would be of equal accuracy, but is objectionable from the fact that, in general, this volume would differ considerably from the arerage volume obtained when $n$ has a greater value (always an integer), and the resulting series of errors would assume larger numerical values.

The errors or corrections are, for purposes of interpolation, most conveniently represented graphically by a smooth curve through points with abscissas proportional to the direct readings

$$
\mathrm{A}, \mathrm{A}+u^{\prime}, \mathrm{A}+u^{\prime}+u^{\prime \prime}, \& \mathrm{c} .
$$

and ordinates to the corresponding corrections.

Should it be necessary to increase the accuracy by a second calibration with a thread of different length, it is only necessary to take one of approximately an integral part of $(B-A)$, and when the final curve of error is drawn make the error at B equal to zero, distributing the difference at that point proportionally to the scale-readings among the errors at the intermediate points-in other words, to shift the axis of the second curve of error so that it shall make the error at $B$ zero.

This method requires for each calibration the use of but a single thread. The computation is simple, and inrolves a minimum of approximation. Errors of obserration are largely eliminated by the number of settings made in all parts of the tube, and by the inspection of the curve of lengths; both of which operations tend in an unusual degree to detect mistakes or any minor irregularities of the capillary. It avoids the common requirements of setting the thread exactly at certain definite points in the tube, or any approximate correction for slight errors in such setting-two sources of considerable error and inconvenience, especially when the thread must be set near or under a line of the graduation. And, lastly, the total time of calibration for a result of given accuracy is reduced to one half or one third of that required by Neumann's method, the quickest and most satisfactory with which I am acquainted except that given by Pickering. The latter, described with some slight inaccuracies, at the reference noted below, is a neat application of the graphical method; and the curve of lengths of thread adopted in the method which I have described is identical with the corresponding one given by Professor Pickering, while the whole process is fully one third shorter and somewhat more accurate. From a series of calibrations executed upon the same thermometer (one with a millimetre-scale, by Baudin, of Paris), using a variety of methods, I have obtained slightly more concordant results with the proposed method than with Neumann's or Pickering's 
(all those possessing, however, nearly the same degree of precision), and decidedly better results with these than with any of the other existing simple methods.

Considerable aid in eliminating errors of parallax in such work is sometimes found by looking down upon the horizontal thermometer through a vertical tube having a small hole at each end. One of the cheap French microscopes with its lenses removed, and inverted in its stand, answers this purpose well.

With such a device two calibrations of the above-described thermometer with threads of 3 and 5 centim. respectively, each with only one series of observations, and requiring not more than one hour and a half each for completion, gave results whose average difference from each other at nine points was 0.04 millim., and the arithmetical sum of the extreme differences was 0.12 millim., a result of sufficient accuracy for any class of work of which such an instrument is capable.

For brief descriptions of methods of separating threads of mercury for calibration, reference may be made to the paper by Russell, and the text-book by Pickering, noted below. These processes are in general use, and are safe and convenient.

References upon Calibration of Closed Thermometer-Tubes.

Bessel, Pogg. Ann. vi. p. 287 (1826).

Rudberg, Pogg. Ann. ix. pp. 353, 566; xxxvii. p. 376 (1836); xl. pp. 39, $562(1837)$.

Kohlrausch, 'Physical Measurements,' p. 59 (English translation).

Pickering, 'Physical Manipulation,' ji. p. 75 (1876).

Thiesen (Neumann's Meth.), Carl's Rep. xv. p. 285 (1879).

Russell (Neumann's Meth., transl. from Thiesen), Amer. Journ. Sci. xxi. p. 373 (1881).

Marek, Carl's Repertorium, xv. p. 300 (1879). (Solution by least squares.)

ron Oettingen, Inaug. Diss., Dorpat, 1865. ('l'his I have been unable to obtain.-S. W. H.)

XXXIV. On Boltzmann's Theorem on the average Distribution of Energy in a System of Material Points*.

To the Editors of the Philosophical Magazine and Journal. Gentlemen, Gräz, April 6, 1882.

DOSSIBLY you may feel disposed to have the accompanying notice of a paper of Maxwell's translated for your valuable 'Philosophical Magazine.' So far as I know, this excellent paper of Maxwell's has not been reprinted in your Magazine; it may not, therefore, be without interest to your readers that some notice of it should appear, if only as

* Translated from Wiedemann's Beiblätter. 